\title{
Ärzte als Vorbilder für ihre Patienten?
}

\author{
Winfried Suske ${ }^{a}$, \\ Walter Kaiser ${ }^{\text {, }}$ \\ Franco Salomon ${ }^{b}$, \\ Verena A. Briner ${ }^{c}$ \\ a Dr. Schlegel Healthworld AG, \\ Steinhausen \\ b Chefarzt Klinik für Innere \\ Medizin, Spital Lachen, \\ Vorstand SGIM \\ c Chefärztin Medizinische Klinik, \\ Kantonsspital Luzern, \\ Präsidentin SGIM
}

Interessenverbindungen und mögliche Interessenkonflikte: keine.

Korrespondenz:

Prof. Dr. med. Verena A. Briner

Kantonsspital Luzern

Medizinische Klinik

Spitalstrasse

CH-6000 Luzern 16

v.a.briner@bluewin.ch

\section{Zusammenfassung}

Im Hinblick auf die Vorbildfunktion von Medizinern ist von Interesse, wie konsequent sich diese selbst an die Gesundheitsempfehlungen zur Vermeidung kardiovaskulärer Erkrankungen oder Krankheiten wie Diabetes mellitus halten. Ziel der vorliegenden Querschnittsbefragung unter den Teilnehmern der Jahresversammlung der Schweizerischen Gesellschaft für Innere Medizin 2007 in Basel war, anhand einer überwiegend Internisten umfassenden Stichprobe diverse gesundheitsrelevante Verhaltensweisen bei Ärztinnen und Ärzten zu erfassen. Angaben zur Soziodemographie, zu Körpergewicht und -grösse sowie zu den vermeidbaren Risikofaktoren Rauchen und Bewegungsmangel wurden in Form eines selbständig auszufüllenden Fragebogens erhoben. Die Ergebnisse lassen den Schluss zu, dass sich die in der vorliegenden Arbeit erfassten Ärztinnen und Ärzte in vielerlei Hinsicht gesundheitsbewusster verhalten als die erwachsene Schweizer Durchschnittsbevölkerung.

\section{Einleitung}

Bereits moderate Änderungen der Lebens- und Ernährungsgewohnheiten beeinflussen das Risiko des Auftretens von Herz-Kreislauf-Krankheiten und Diabetes mellitus günstig [1]. Wissenschaftliche Studien zeigen, dass das persönliche Gesundheits- bzw. Risikoverhalten von Ärzten mit darüber entscheidet, mit welcher Konsequenz sie ihre Patienten über Gesundheitsrisiken und eine gesündere Lebensführung aufklären und beraten [2-5]. Bekannt ist, dass Ärzte, die selbst rauchen, Patienten mit grösserer Zurückhaltung nach dem Rauchverhalten fragen bzw. zur Aufgabe des Tabakkonsums ermuntern. Die Meinung des Arztes hat einen grossen Einfluss auf die Motivation aufhörwilliger Raucher [6]. Andererseits nehmen Patienten die Aufforderungen zur Gewichtsabnahme offenbar weniger ernst, wenn sie von übergewichtigen oder adipösen Ärzten erteilt werden [7]. Zur Häufigkeit von Übergewicht in der Schweizer Ärzteschaft liegen unseres Wissens bislang keine Daten vor.

\section{Methoden}

An die Teilnehmer der 75. Jahresversammlung der Schweizerischen Gesellschaft für Innere Medizin (SGIM) in Basel wurde ein strukturierter, in

\section{Questionnaire sur le comporte-}

ment à l'égard de la santé

des internistes suisses:

les médecins sont-ils des modèles pour leurs patients?

Concernant la fonction de modèle exercée par les médecins, il paraît intéressant de savoir à quel point ceux-ci suivent eux-mêmes les conseils de santé visant à éviter les maladies cardio-vasculaires ou le diabète. L'objectif de la présente enquête transversale effectuée auprès des participants à l'assemblée annuelle de la Société Suisse de Médecine Interne 2007 à Bâle était de relever chez les médecins, à l'aide d'un échantillon comprenant essentiellement des internistes, plusieurs comportements en matière de santé. Les données saisies, sous forme d'un questionnaire à remplir de manière autonome, portaient sur la sociodémographie, le poids corporel et la stature ainsi que sur les facteurs de risques évitables tels que le tabagisme et la sédentarité. Les résultats concluent que les médecins figurant dans le présent travail sont à maints égards plus conscients de leur santé que la population adulte suisse dans son ensemble.

deutscher und französischer Version erstellter Fragebogen zur Soziodemographie und zu gesundheitsrelevanten Lebensgewohnheiten abgegeben. Von 2200 verteilten Fragebögen wurden im Laufe der drei Kongresstage 497 Fragebögen ausgefüllt retourniert (Rücklauf 23\%), von denen 493 zur Auswertung herangezogen werden konnten. Die vollständig anonymisierten Daten wurden in Microsoft Excel erfasst und mit monovariaten Analysemethoden ausgewertet.

\section{Ergebnisse und Diskussion}

Die Angaben zur Soziodemographie und Arbeitssituation der befragten Ärztinnen und Ärzte sind in Tabelle 1 zusammengefasst. Das Durchschnittsalter der Befragten liegt bei $44 \pm 10$ Jah- 
Tabelle 1

Soziodemographie der an der Jahresversammlung 2007 der Schweizerischen Gesellschaft für Innere Medizin (SGIM) befragten Ärztinnen und Ärzte.

\section{Geschlechtsverteilung \\ Frauenanteil (\%)}

Alter $(n=493)$

Mittelwert in Jahren (Stabw.)

$44,1(10,3)$

Häufigkeit nach Altersklassen (\%)

$<35$ Jahre

24,5

36-45 Jahre

31,4

46-55 Jahre

28,8

56-65 Jahre

13,8

$>65$ Jahre

1,4

Sprachregion (\%) $(n=493)$

\section{Deutschschweiz}

\section{6}

Westschweiz

22

Tessin

Arbeitsort (\%) $(n=472)$

Praxis

40

Spital

48

Praxis und Spital

11

sonstiges

Fachrichtung (\%) $(n=488)$ (nicht gleichbedeutend mit FA-Titel)

Innere Medizin

83,2

Allgemeinmedizin

Anteil Assistenzärzte (\%)

treffen sind: Wie in der Gesamtbevölkerung sind auch in der Berufsgruppe der Ärzte deutlich weniger Frauen übergewichtig als Männer (10\% vs. $27,5 \%$ ). Wie aus Abbildung 1 ersichtlich, ist mit höherem Alter der Umfrageteilnehmer ein Anstieg des mittleren Körpergewichts zu beobachten. Dabei bestätigt sich das Phänomen, dass bei Frauen der BMI im Laufe des Lebens kontinuierlich zunimmt, während sich bei Männern das Maximalgewicht früher einstellt, im vorliegenden Fall in der Altersgruppe der 36- bis 45jährigen. Bei den in der Schweizerischen Gesundheitsbefragung 2002 erfassten Männern wird das Plateau in der Gruppe der 45- bis 54jährigen erreicht.

\section{Rauchen}

Die Erhebung des Raucherstatus ergab, dass derzeit 8,7\% der befragten Ärzte rauchen (Tab. 3). Dabei raucht etwa ein Drittel von ihnen (14 von 43 Frauen und Männern) regelmässig, der Rest gelegentlich. Abbildung 2 zeigt, dass Ärztinnen eine leicht grössere Nikotinabstinenz erkennen lassen als ihre männlichen Kollegen, dass jene aber beim Gelegenheitsrauchen mit diesen mithalten. Während die Raucherinnen und Raucher gleichmässig über alle Altersgruppen verteilt sind, sind die Gelegenheitsraucher mit einer Häufigkeit von $44 \%$ vor allem in der Altersgruppe der 36- bis 45 jährigen zu finden (nicht gezeigt). In der mehr als 15 Jahre alten Schweizer Gesamtbevölkerung liegt die Raucherquote bei $31 \%$, was bedeutet, dass die befragten Ärzte etwa viermal seltener rauchen als der Schweizer Durchschnittsbürger.

Die Daten bestätigen den bereits in anderen Studien beobachteten Trend, dass der Anteil der Nichtraucher unter den Medizinern in der Schweiz wächst. Gemäss einer im Jahr 2002 durchgeführten Umfrage unter 1784 Schweizer Hausärzten (regionale Verteilung: 71,6\% Deutschschweiz, 24,5\% Westschweiz, 3,9\% Tessin) bezeichneten sich $12,6 \%$ der männlichen und $11 \%$ der weiblichen Kollegen als Raucher [9]. Andere Autoren hatten in Querschnittsbefragungen von Schweizer Ärzten Raucherquoten von $20 \%$ ermittelt [2, 10]. Eine zwischen 1955 und 1973 zur Mortalität Schweizer Ärzte infolge Rauchens durchgeführte Kohortenstudie ergab, dass der Anteil der Raucher im genannten Zeitraum von 37 auf 21\% zurückgegangen ist [11]. Laut dieser Studie rauchten vor allem die Internisten - neben Pneumologen und Hals-Nasen-Ohren-Ärzten - weniger als andere Ärzte. Die mit 1,6\% bislang niedrigste unter Medizinern ermittelte Raucherquote stammt von einer zu 93 \% männlichen Gruppe US-ameUnterschiede zwischen den Geschlechtern anzu$30 \mathrm{~kg} / \mathrm{m}^{2}$ ), 8,6\% stark übergewichtig bzw. adipös (BMI $>30 \mathrm{~kg} / \mathrm{m}^{2}$ ). Aus Tabelle 2 ist ersichtlich, dass bei der Häufigkeitsverteilung nach BMI- ren. Rund $83,2 \%$ der Umfrageteilnehmer ge-

Rund drei Viertel (73\%) der befragten Ärztinnen unde sind gemäss ihren eigenen Angaben gewichtig anzusehen (BMI zwischen 19 und $25 \mathrm{~kg} / \mathrm{m}^{2}$ ). Laut Bericht zur Schweizerischen für 48,7\% der Bevölkerung zu [8]; 32,7\% der Schweizer im Alter von 25 bis 74 Jahren sind dagegen übergewichtig (BMI zwischen 25 und 
Tabelle 2

Body-Mass-Index (BMI). Ergebnisse der unter den Ärztinnen und Ärzten der SGIM-Jahresversammlung 2007 durchgeführten Umfrage und des Vergleichskollektivs der Schweizerischen Gesundheitsbefragung 2002 [8].

\begin{tabular}{|c|c|c|}
\hline & SGIM-Umfrage Basel 2007 & Gesundheitsbefragung Schweiz 2002 \\
\hline Mittelwert Body-Mass-Index (BMI) & in $\mathrm{kg} / \mathrm{m}^{2}$ (Stabw.) & \\
\hline Ärztinnen/Ärzte gesamt ( $n=492)$ & $23,3(2,8)$ & \\
\hline$w(n=180)$ & $22,1(3,0)$ & \\
\hline$m(n=313)$ & $23,9(2,6)$ & \\
\hline Häufigkeitsverteilung nach BMI-Klassen & in $\%(w / m)$ & in $\%(w / m)$ \\
\hline $\mathrm{BMI}<19$ & $3,5(8,3 / 0,6)$ & $10,0(16,7 / 2,8)$ \\
\hline $19 \leq \mathrm{BMI}<25$ & $73,0(79,4 / 69,3)$ & $48,7(50,7 / 46,5)$ \\
\hline $25 \leq \mathrm{BMI}<30$ & $21,1(10,0 / 27,5)$ & $32,7(24,3 / 41,8)$ \\
\hline $\mathrm{BMI} \geq 30$ & $2,4(2,2 / 2,6)$ & $8,6(8,2 / 9,0)$ \\
\hline
\end{tabular}

Tabelle 3

Rauchverhalten. Ergebnisse der unter den Ärztinnen und Ärzten der SGIM-Jahresversammlung 2007 durchgeführten Umfrage und des Vergleichskollektivs der Schweizerischen Gesundheitsbefragung 2002 [8].

\begin{tabular}{lll} 
& SGIM-Umfrage Basel 2007 & Gesundheitsbefragung Schweiz 2002 \\
\hline Häufigkeiten & in $\%$ & in \% \\
\hline Nichtraucher $(\mathrm{n}=492)$ & 91,3 & \\
\hline w & 93,3 & \\
\hline m & 90,1 & 31 \\
\hline Raucher (davon Gelegenheitsraucher) & $8,7(5,9)$ & 26 \\
\hline w & $6,7(5,6)$ & 36 \\
\hline m & $9,9(6,1)$ & \\
\end{tabular}

rikanischer Kardiologen [12]; von den 471 befragten Ärzten gaben $84 \%$ an, noch nie geraucht zu haben, $14 \%$ hatten früher geraucht.

\section{Körperliche Aktivität/Bewegungsmangel}

Mehr als drei Viertel (81\%) der im Rahmen dieser Arbeit befragten Ärztinnen und Ärzte gaben an, mindestens einmal in der Woche Sport zu treiben oder sich während 30 Minuten intensiv zu bewegen (Tabelle 4). Mit dreimal in der Woche Sport oder intensiver Bewegung sind rund ein Drittel der Befragten in ihrer Freizeit bewegungsmässig aktiv, knapp 4\% der Ärzte täglich. Körperlich inaktiv sind dagegen 7,3\%. Direkte Vergleiche zwischen der Gruppe der Ärzte und der Bevölkerung werden durch die Tatsache erschwert, dass körperliche Aktivität und Bewegungsmangel anhand unterschiedlicher Kriterien und Skalen definiert werden $[8,13]$. Anhand eines fünfstufigen Leitindikators ist laut Schweizerischer Gesundheitsbefragung je ein Fünftel der Menschen in der Freizeit völlig inaktiv bzw. teilaktiv. Gesundheitsrelevant sind dabei nur körperliche Aktivitäten, die mindestens zu Ausser-Atem-Kommen oder (leichtem) Schwitzen führen [14]. Nach einer dreistufigen Skala, die auch die Erfüllung wichtiger Bewegungsempfehlungen berücksichtigt, gelten 35,9\% der Schweizer als aktiv und $44,7 \%$ als teilaktiv. Nach beiden Skalen werden 19,4\% der Schweizer als Bewegungsmuffel angesehen, zweieinhalbmal mehr als in der hier präsentierten Stichprobe Schweizer Internisten. Eine Studie mit 496 Hausärzten dreier Westschweizer Kantone hatte ergeben, dass sich 57\% der Befragten immerhin dreimal im Monat oder mehr für die Zeitdauer von mindestens 20 Minuten sportlich betätigen. Bei 21\% war dies eigenen Angaben zufolge weniger als einmal im Monat bzw. nie der Fall [6].

\section{Schlussfolgerung}

Insgesamt geben sich die im Rahmen der vorliegenden Befragung erfassten Ärztinnen und Ärzte gesundheitsbewusster als das Gros der Schweizer Bevölkerung. Vorbildlich sind die befragten Mediziner, mehrheitlich Internisten, vor allem 


\section{Abbildung 1}

BMI-Verteilung nach Altersgruppen und Geschlecht. Bei der Gesamtgruppe ist zusätzlich die Standardabweichung angegeben. Ergebnisse der unter den Ärztinnen und Ärzten der SGIM-Jahresversammlung 2007 durchgeführten Umfrage.

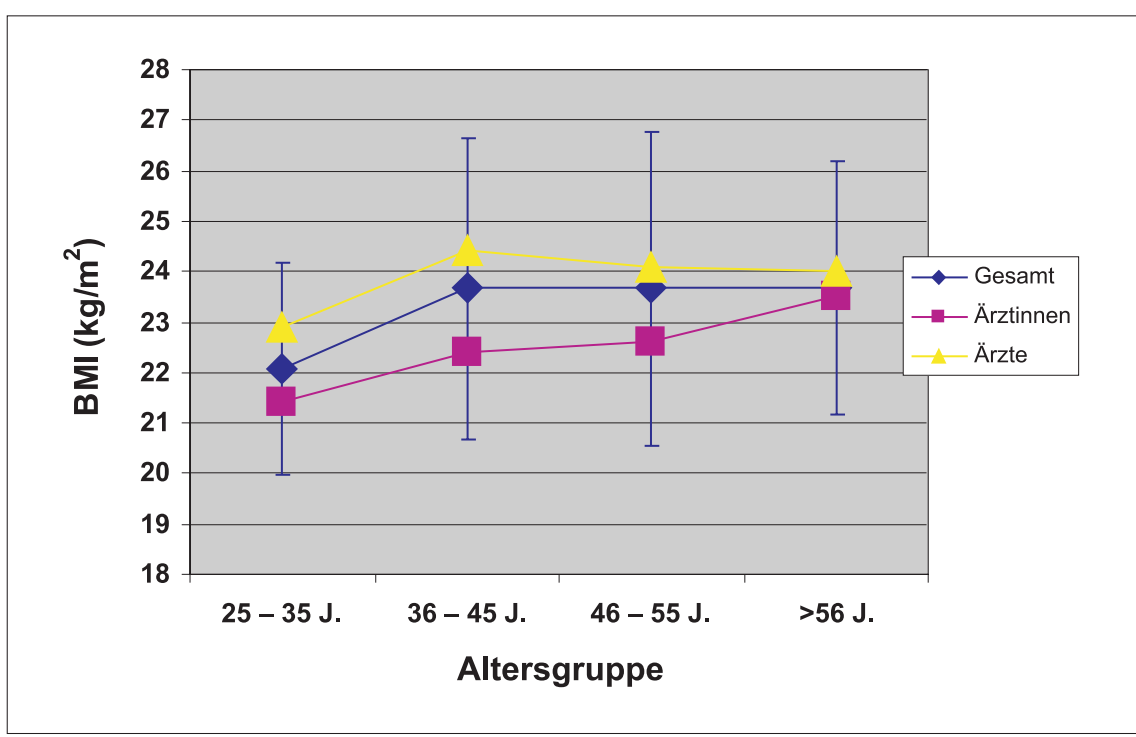

\section{Abbildung 2}

Geschlechterunterschied beim Tabakkonsum. Ergebnisse der unter den Ärztinnen und Ärzten der SGIM-Jahresversammlung 2007 durchgeführten Umfrage.

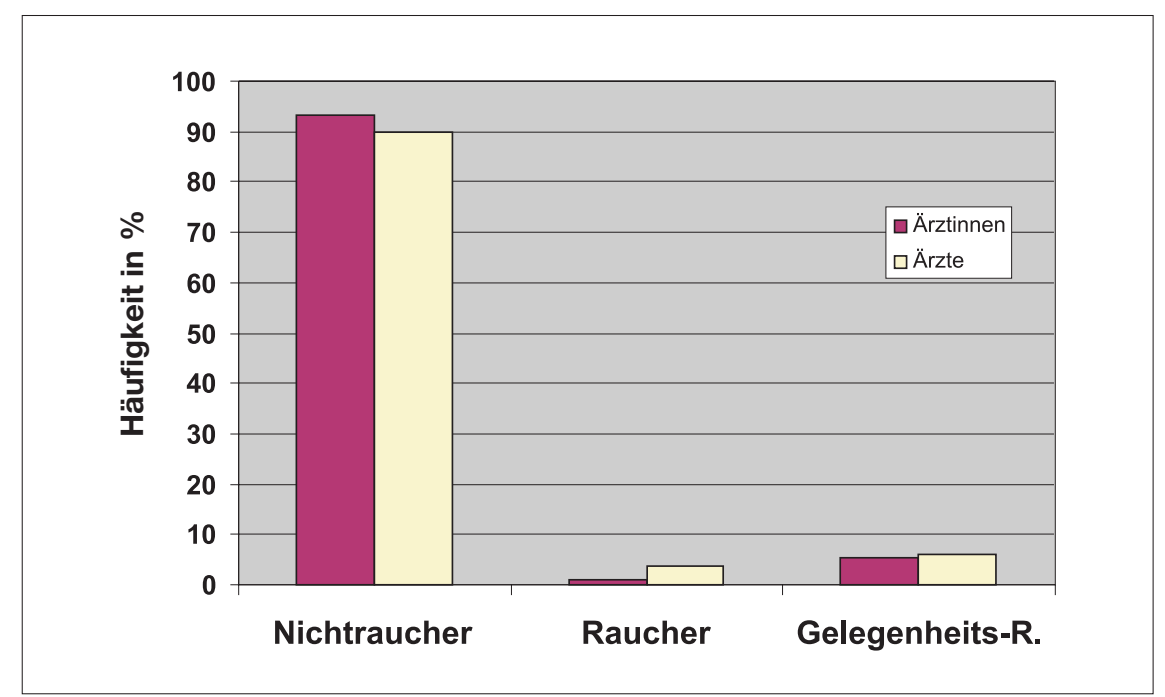

\section{Tabelle 4}

Körperliche Aktivität in Form von Sport oder intensiver Bewegung von mindestens 30 Minuten. Ergebnisse der unter den Ärztinnen und Ärzten der SGIM-Jahresversammlung 2007 durchgeführten Umfrage $(n=492)$.

\section{Umfang der aktiven Betätigung}

nie

in $\%(\mathrm{w} / \mathrm{m})$

mind. $1 \mathrm{mal}$ pro Woche

$7,3(8,3 / 7,0)$

mind. 2 mal pro Woche

$81,1(77,8 / 82,7)$

$65,7(61,1 / 68,1)$

$33,1(31,1 / 34,2)$

mind. $3 \mathrm{mal}$ pro Woche

$3,9(3,3 / 4,2)$ beim Rauchen und beim Halten des Normalgewichtes: Die Prävalenz des Rauchens ist in der Gruppe der Ärzte 4mal geringer als in der Gesamtbevölkerung, die des Übergewichts $1,5 \mathrm{mal}$ bei den Männern bzw. 2,5mal geringer bei den Frauen.

Im Hinblick auf die Bedeutung der Unterschiede beim Gesundheitsverhalten beider Gruppen sei angemerkt, dass möglicherweise ein Teil der Umfrageteilnehmer dazu neigte, einzelne Antworten den aus sozialer und beruflicher Sicht wünschenswerten Ergebnissen anzupassen. Denkbar ist auch, dass sich tendenziell Kollegen und Kolleginnen an der Umfrage beteiligten, die stärker auf ihre eigene Gesundheit achten als diejenigen, die sich nicht beteiligten. Ein Hinweis hierauf könnte das im Vergleich zu anderen Untersuchungen zur Ärztegesundheit niedrige Durchschnittsalter sein. Zusätzlich sei darauf hingewiesen, dass sich die Gruppe der 493 befragten Ärzte und die Vergleichsgruppe, die Teilnehmer der Schweizerischen Gesundheitsbefragung, mit grosser Wahrscheinlichkeit beim sozioökonomischen Status, beim Bildungsstand und bei der Alterszusammensetzung unterscheiden. Eventuell hat sich auch das Gesundheitsverhalten der Bevölkerung seit Erhebung der Daten im Jahr 2002 verändert.

Mit der mehrheitlichen Beachtung gängiger Gesundheitsempfehlungen zu den drei wichtigsten beeinflussbaren kardiovaskulären Risikofaktoren erfüllen die Schweizer Ärztinnen und Ärzte jedenfalls eine wichtige Voraussetzung, um von der Bevölkerung als Vorbilder für die eigene Gesundheitsvorsorge wahrgenommen $\mathrm{zu}$ werden.

\section{Literatur}

1 Knoops KT, de Groot LC, Kromhout D, Perrin AE, Moreiras-Varela O, Menotti A, van Staveren WA. Mediterranean diet, lifestyle factors, and 10-year mortality in elderly European men and women: the HALE project. JAMA. 2004;292(12):1433-9.

2 Eckert T, Junker C. Motivation for smoking cessation: what role do doctors play? Swiss Med Wkly. 2001;131(35-36):521-6.

3 Wells KB, Lewis CE, Leake B, Ware JE Jr. Do physicians preach what they practice? A study of physicians' health habits and counseling practices. JAMA. 1984;252(20):2846-8.

4 Frank E, Bhat Schelbert K, Elon L. Exercise counseling and personal exercise habits of US women physicians. J Am Med Womens Assoc. 2003; 58(3):178-84.

5 Abramson S, Stein J, Schaufele M, Frates E, Rogan S. Personal exercise habits and counseling practices of primary care physicians: a national survey. Clin J Sport Med. 2000;10(1):40-8. 
6 Cornuz J, Ghali WA, Di Carlantonio D, Pecoud A, Paccaud F. Physicians' attitudes towards prevention: importance of intervention-specific barriers and physicians' health habits. Fam Pract. 2000; 17(6):535-40.

7 Hash RB, Munna RK, Vogel RL, Bason JJ. Does physician weight affect perception of health advice? Prev Med. 2003;36(1):41-4.

8 Gesundheit und Gesundheitsverhalten in der Schweiz 1992-2002. Schweizerische Gesundheitsbefragung. Neuenburg: Bundesamt für Statistik; 2005. www.statistik.admin.ch.

9 Sebo P, Bouvier Gallacchi M, Goehring C, Künzi B, Bovier PA. Use of tobacco and alcohol by Swiss primary care physicians: a cross-sectional survey. BMC Public Health. 2007;7:5

10 Grüninger U, Mion H, Abelin T. Immer weniger Ärzte sind Raucher. Schweiz Ärztezeitung. 1990; 71:739-43.
11 Gsell O, Abelin T, Wieltschnig E. Smoking and mortality in Swiss physicians: results of an 18-year survey. Bull Schweiz Akad Med Wiss. 1979; 35(1-3):71-82.

12 Abuissa H, Lavie C, Spertus J, O'Keefe J. Personal health habits of American cardiologists. Am J Cardiol. 2006;97(7):1093-6.

13 Lamprecht M, Stamm H. Bewegung, Sport, Gesundheit. Fakten und Trends aus den Schweizerischen Gesundheitsbefragungen 1992, 1997 und 2002. Neuenburg: Bundesamt für Statistik; 2006.

14 Bundesamt für Sport (BASPO), Bundesamt für Gesundheit (BAG), Gesundheitsförderung Schweiz und Netzwerk Gesundheit und Bewegung Schweiz Gesundheitswirksame Bewegung. Ein Grundsatzdokument. Magglingen: Bundesamt für Sport; 2002. 